\title{
Tratamiento farmacológico para pacientes con diabetes
}

\section{Pharmacological treatment for patients with diabetes}

\section{Tratamento farmacológico para pacientes com diabetes}

\author{
Mary I. Vinces-Zambrano I \\ mary.vinces@gmail.com \\ Patricia M. Espinel-Zambrano II \\ espinel.paty@hotmail.com \\ Andrea N. Pico-Tagle III \\ andrepico@gmail.com \\ Sarai E. Del Castillo Pavón IV \\ sari178@yahoo.es \\ Gloria E. Chávez De La Torre ${ }^{\mathrm{V}}$ \\ gloria-chavez@hotmail.es \\ Edwin D. Betancourth-Justicia VI \\ edwin1785@gmail.com
}

Recibido: 27 de junio de 2018 * Corregido: 28 de agosto de 2018 * Aceptado: 20 de septiembre de 2018

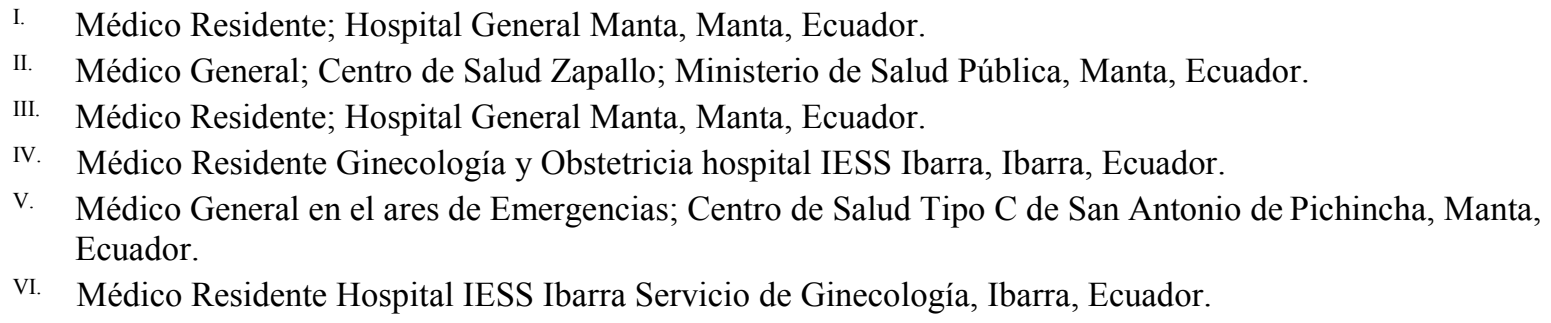

VI. Médico Residente Hospital IESS Ibarra Servicio de Ginecología, Ibarra, Ecuador. 


\section{Resumen}

En el presente artículo se estudia la importancia del tratamiento farmacológico para pacientes con diabetes, puesto que en la terapia varios son los motivos por los cuales estudiar la adherencia a los tratamientos con los medicamentos. En primera instancia, parte de las indicaciones terapéuticas farmacológicas no pueden ser controladas diariamente por un funcionario externo al paciente por el contrario, quedan bajo su absoluta responsabilidad, por lo tanto, es el paciente quien terminantemente decide si cumple o no. En consecuencia, las bajas tasas de adherencia comunicadas, consideran afirmar la idea de que el paciente mayoritariamente no cumple con el tratamiento de la diabetes, las recomendaciones deben estar basadas con el fin de contribuir al control metabólico y reducir las complicaciones vasculares, acrecentando la calidad y el estilo de vida de los pacientes, se establece actualmente considerar el nivel de obesidad así como el estado de la actividad física del paciente, incorporado al tratamiento farmacológico. Si un paciente no cumple con la prescripción médica es improbable estimar el efecto del tratamiento farmacológico, lo que a su vez puede contribuir a extender la terapia y mantener enfermedades o problemas de salud que incluso pueden conducir a la muerte, con el inmediato costo económico para el estado, referente a materias concernientes a pérdida de productividad y costos asociados a rehabilitación y pensiones de invalidez. Es necesario enfatizar al paciente la aceptación de su padecimiento e identificar los trastornos afectivos y de ansiedad que ello implica, ya que su manejo adecuado también se asocia con una mejoría en la calidad de vida y en el apego terapéutico. La trascendencia económica del consumo inadecuado de fármacos es indiscutible y es un auténtico reto para los administradores, debido al derroche económico que hacen los pacientes.

Palabras claves: Tratamiento farmacológico; adherencia a los tratamientos; estilo de vida; reducir las complicaciones; costo económico. 


\begin{abstract}
In the present article the importance of pharmacological treatment for patients with diabetes is studied, since in therapy there are several reasons for studying the adherence to treatments with medications. In the first instance, part of the pharmacological therapeutic indications can not be controlled daily by an official external to the patient; on the contrary, they remain under their absolute responsibility, therefore, it is the patient who strictly decides whether or not to comply. Consequently, the low adherence rates reported, affirm the idea that the patient mostly does not comply with the treatment of diabetes, the recommendations must be based in order to contribute to metabolic control and reduce vascular complications, increasing the quality and the lifestyle of the patients, it is currently established to consider the level of obesity as well as the state of the patient's physical activity, incorporated into the pharmacological treatment. If a patient does not comply with the medical prescription it is unlikely to estimate the effect of pharmacological treatment, which in turn may contribute to extend the therapy and maintain diseases or health problems that may even lead to death, with the immediate economic cost to the state, referring to matters concerning loss of productivity and costs associated with rehabilitation and disability pensions. It is necessary to emphasize the patient's acceptance of their condition and identify the affective and anxiety disorders that this implies, since their proper management is also associated with an improvement in the quality of life and therapeutic attachment. The economic importance of inadequate drug use is indisputable and is a real challenge for administrators, due to the economic waste that patients make.
\end{abstract}

Key words: Pharmacological treatment; adherence to treatments; lifestyle; reduce complications; economic cost. 


\section{Introducción.}

La diabetes mellitus (DM) es una enfermedad cuya prevalencia mundial se ha incrementado en los últimos años. Con un importante número de casos que persisten sin diagnosticar. $\mathrm{Su}$ prevalencia es mayor en hombres y aumenta con la obesidad, la edad, presencia de HTA, elevado nivel de triglicéridos, bajo nivel de HDL-colesterol, bajo nivel de estudios y antecedentes familiares de diabetes. El sobrepeso y la obesidad son los factores de riesgo más importantes asociados con inactividad física y alimentación inadecuada.

Se estima que más de 371 millones de personas en el mundo padecen diabetes mellitus (DM), una cifra que aumenta anualmente y que produce cada año 4,8 millones de muertes debidas a complicaciones derivadas de la enfermedad, WHO, (2013).

Frente la capacidad de estas cifras, es de vital importancia lograr los objetivos de los tratamientos planteados a fin de evitar las graves complicaciones unidas a esta enfermedad, por lo que es crítico en estos pacientes conseguir una adecuada adherencia al tratamiento. El cumplimiento en el tratamiento se define como la disposición que el paciente asume de acuerdo con las normas o consejos proporcionados por el médico, enfermero o personal de salud, de acuerdo con los hábitos o estilo de vida recomendados, tanto así como del propio tratamiento farmacológico prescrito.

Estas normas y consejos son realizadas tras una decisión completamente razonada, por parte del paciente, abandonándose las connotaciones de sumisión que este término implicaba para el paciente, Márquez y Casado, (2001).

El incumplimiento terapéutico del tratamiento en la diabetes mellitus tipo 2 (DM2) ha sido escasamente estudiado existiendo pocos trabajos que lo analicen. Es una de las principales causas de 
mal control de las glucemias basales y hemoglobina glucosilada, habiéndose observado su asociación a un peor control de la DM2, Cramer, et al. (2008) y Donnelly, et al. (2007).

En la actualidad, ha sido rebasado el concepto que identificaba a la diabetes como el descontrol de la glucemia. Ahora se sabe que se trata de una alteración metabólica en la cual, además de la glucosa, se encuentran involucrados los lípidos. No obstante, en la práctica médica cotidiana la piedra angular para evaluar el control de la diabetes continúa siendo el estado de la glucosa en sangre. Si bien las cuantificaciones sanguíneas muestran el comportamiento día a día, no ofrecen la posibilidad de conocer la evolución en el mediano plazo; en este caso, el uso de la hemoglobina glucosilada es la mejor opción, Goldstein, et al. (2004) y Rush \& O’Connor, (2002).

Las personas con DM2 deberán recibir las indicaciones para el manejo integral de la enfermedad por parte de un equipo multidisciplinario de salud coordinado por el médico. Tal equipo incluye, pero no está limitado, al médico, la enfermera, la dietista y los profesionales en salud mental con experiencia y especial interés en diabetes, Matsuoka, (2001).

Es esencial que este equipo proporcione la orientación con un enfoque tal que el paciente asuma un papel activo en el cuidado de la enfermedad. El tratamiento integral para la educación al paciente y su familia debe incluir la orientación en forma sencilla respecto a lo que es la enfermedad, las características de la alimentación y el ejercicio que debe realizarse; el tratamiento debe estar fundamentado en la vigilancia de las condiciones clínicas y metabólicas y la prescripción apropiada de los medicamentos, American Diabetes Association, (2002).

Aun cuando se sabe que la prescripción —entendida como la indicación proporcionada por el profesional de la salud al diabético — es fundamental en la búsqueda del control metabólico, esto no 
es suficiente. Lo que se conoce es que la participación del paciente y su núcleo familiar tiene un papel protagónico en el apego al tratamiento prescrito, Kim, Oh, (2003) y Martín, Rodríguez, (2001).

La irrupción de nuevas tecnologías y tratamientos farmacológicos han posibilitado contar cada día con mejores y más variadas prescripciones médicas. Esto, a su vez, debiera significar un aumento tanto en la efectividad de los tratamientos, así como también incidir directamente en la calidad de vida de los pacientes. Sin embargo, para que esto ocurra debieran existir al menos dos condiciones: una adecuada prescripción por parte del equipo médico y un correcto cumplimiento de la misma, Ortiz y Ortiz, (2007).

\section{Metodología.}

El presente trabajo científico tiene una visión directamente específica, se hizo la revisión de literatura más próxima. Para la realización de la actual investigación, considerando, información confiable sobre trabajos figurados, versados en este tema, se utilizaron informaciones bibliográficas, se la obtuvo de libros electrónicos, consultas electrónicas, artículos científicos y revistas.

\section{Desarrollo.}

\section{La diabetes}

La diabetes es un desorden metabólico que responde a múltiples etiologías y afecta a varios órganos del cuerpo humano La cuantificación de la presencia de diabetes a partir de la medición de glucosa (forma de azúcar simple que sirve como materia prima para la composición de carbohidratos) es un indicativo de la carga que los servicios de salud tendrán que asumir por esta 
condición La ENSANUT-ECU tomó como criterio diagnóstico de diabetes el valor de glucosa medida en suero (glucemia) con un período de ayunas de por lo menos ocho horas, a partir de 126 mg/dl ('Standards of Medical Care in Diabetes-2012', 2012), citado por Freire, et al., (2014).

Las consecuencias de estas prácticas alimentarias y sedentarismo se presentan en los resultados obtenidos en la aproximación a las enfermedades crónicas y en las tasas de sobrepeso u obesidad presentadas anteriormente. La diabetes mellitus junto a la dislipemia y la hipertensión arterial constituyen los padecimientos crónicos no transmisibles que registran la mayor cantidad de consultas y egresos hospitalarios desde hace más de dos décadas. A partir de la tercera década de vida, comparada con la segunda década, la prevalencia de diabetes se cuadruplica, y entre las edades de 30 y 50 años se quintuplica. Freire, et al., (2014).

El impacto de la falta de adherencia terapéutica en los pacientes diabéticos ha sido estudiado anteriormente utilizando diversas metodologías. En un registro publicado en el año 2012 se puso de manifiesto que solo el 56\% de los pacientes con DM2 lograron el control metabólico, a pesar de que aproximadamente el $78 \%$ de los pacientes seguía tratamiento con antidiabéticos orales o con insulina, Vinagre, et al. (2012).

La falta de apego al tratamiento implica grandes erogaciones económicas, ya que más de $10 \%$ de los ingresos hospitalarios se deben a esta causa.5 Los factores de riesgo para el no apego terapéutico son los relacionados con el paciente, la enfermedad, el médico tratante, el lugar donde se prescribe el tratamiento y el medicamento en sí. Los estudios sobre este tema muestran que es de $50 \%$ de cumplimiento en enfermedades crónicas; 6 y en enfermedades agudas, como la diarrea, varía entre $31 \%$ y $49 \%$, con los siguientes factores de riesgo asociados: desconocimiento de la enfermedad, desconfianza de la capacidad del médico, duración de la consulta menor a cinco 
minutos, falta de comprensión de las indicaciones médicas, escolaridad baja, estado civil e intolerancia a los medicamentos, Durán, et al. (2001).

Al igual que otros autores consideran en el caso de otras enfermedades crónicas, el incumplimiento terapéutico constituye un problema sanitario de primer orden que afecta directamente a los pacientes, independientemente de su edad y de su situación clínica. En primer lugar reduce la posibilidad de alcanzar el control metabólico adecuado aumentando la morbimortalidad, lo que ya ha sido demostrado en otros estudios: un incremento de la adherencia a los antidiabéticos orales del $10 \%$ supone un descenso de la HbAlc del $0,1 \%$. Este mal control se asocia a un aumento de las hospitalizaciones debidas a complicaciones y reduce la calidad de vida del paciente, siendo ambos hechos conocidos por los expertos consultados en el presente estudio, Orozco, et al. (2016).

Es necesario enfatizar al paciente la aceptación de su padecimiento e identificar los trastornos afectivos y de ansiedad que ello implica, ya que su manejo adecuado también se asocia con una mejoría en la calidad de vida y en el apego terapéutico. La trascendencia económica del consumo inadecuado de fármacos es indiscutible y es un auténtico reto para los administradores, debido al derroche económico que hacen los pacientes; en estudios realizados con este propósito, se encontró un consumo menor a 75\%. Durán, et al. (2001).

Existe acuerdo en considerar la importancia de la medición periódica del cumplimiento terapéutico, ya que hay distintas modalidades de incumplimiento farmacológico, en función de cuándo decide el paciente dejar de tomar la medicación. Por un lado, es posible que el paciente ni siquiera comience a tomar la medicación, o puede comenzar a tomarla del modo adecuado pero al cabo de un tiempo abandonarla prematuramente. Además, el cumplimiento puede variar en el 
tiempo, tal y como se ha descrito en otros estudios, observándose un descenso del porcentaje de pacientes cumplidores, que pasa del $82,5 \%$ en los primeros 6 meses de tratamiento al $71,8 \%$ en los últimos 6 meses en pacientes con DM2 que usaban insulina, Orozco, et al. (2016).

La educación del paciente es un factor clave que debe ser combinada con intervenciones motivacionales centradas en producir cambios significativos en el comportamiento del paciente con relación a su enfermedad y su tratamiento. Sin embargo, los resultados del consenso muestran que los profesionales sanitarios no suelen disponer de tiempo suficiente en sus consultas para trabajar estos aspectos. Facilitar asesoramiento por parte de una enfermera, fomentar el papel activo del farmacéutico, proporcionar al paciente materiales educativos, estrategias para favorecer el autocontrol del paciente y herramientas que ayuden a disminuir el número de olvidos, como mensajes de texto, recordatorios en los móviles o programas a través de un contact center para apoyar al paciente bajo la supervisión del personal sanitario, han demostrado ser eficaces para mejorar la adherencia terapéutica, Orozco, et al. (2016).

Se ha observado un porcentaje de incumplidores del tratamiento con insulina en la DM2 del $25,25 \%$, observándose un descenso significativo a lo largo del seguimiento, siendo del $82,5 \%$ en los primeros 6 meses y del 71,8\% en los últimos 6 . El porcentaje de cumplimiento medio fue del 90,9\%, con un descenso no significativo a lo largo del seguimiento. Estos descensos a lo largo del seguimiento han sido observados en otros estudios de investigación sobre cumplimiento en otras enfermedades, con visitas iniciales más cercanas en el tiempo, donde se observaron inicialmente incrementos en el cumplimiento a consecuencia del consabido efecto Hawthorne de los estudios de investigación y del efecto pasajero de la posible intervención realizada por el médico en su primera visita. Por consiguiente en nuestro estudio se ha observado como una cuarta parte de los diabéticos 
dejan de administrarse más de un $20 \%$ de la insulina prescrita. Este estudio es el primero realizado en España que analiza el cumplimiento del tratamiento con insulina, con lo cual no hay bibliografía para su comparación, Márquez, et al. (2012).

\section{Tratamiento farmacológico de la diabetes}

Los agentes orales están indicados en quienes fallan las recomendaciones iniciales para llevar a cabo un adecuado control glucémico con la dieta y el ejercicio físico. El estudio de UKPDS ha mostrado que la DM2 es un trastorno progresivo que puede ser tratado en un principio con agentes orales en monoterapia, pero puede requerir la adición de otros agentes orales en combinación; en algunos pacientes la terapia con insulina puede ser necesaria para llevar a cabo el control glucémico, De-Fronzo, (1999).

Según Hernández, et al. (2011), manifiesta que 85\% de la población estudiada usa medicamentos, esta variable no se asoció a un mejor control. Esto quizá se deba, primero, a que el uso de medicamentos sólo está justificado cuando el paciente no se controla con dieta y ejercicio, y en segundo lugar, a que los pacientes no suelen acudir al médico sino hasta que empiezan a presentar las complicaciones que resultan de un descontrol metabólico; y finalmente, los pacientes que requieren tomar medicamentos tienen un tiempo de evolución más largo que aquellos que no los toman. Lo anterior resulta alarmante e indica que es urgente revisar a detalle el modelo de atención y la calidad del mismo.

El tratamiento intensivo y adecuado se relaciona con el retardo en la aparición y progresión de las complicaciones crónicas de la enfermedad, por lo que parece razonable recomendar un control estricto de su tratamiento. Se define apego al tratamiento como la conducta del paciente que 
coincide con la prescripción médica, en términos de tomar los medicamentos, seguir las dietas o transformar su estilo de vida. El apego al tratamiento es importante para evaluar la evolución clínica, ya que una alianza terapéutica entre el paciente y el médico es necesaria para el éxito del tratamiento. Existen diversas técnicas para medir el apego al tratamiento farmacológico, como la cuantificación directa del fármaco en sangre u orina, entrevista al paciente y el conteo de tabletas, entre otras, Durán, et al. (2001).

El apego terapéutico ha sido definido como la conducta del paciente que coincide con la prescripción médica, en particular en las enfermedades crónicas, y presenta un amplio rango de variación que va de 30 a $80 \%$, Evans, et al. (2002).

En pacientes con diabetes mellitus tipo 2, obesos o no, el tratamiento con metformina reduce de 1 a $2 \%$ la HbAlc.1 Cuando hay obesidad, el uso de la biguanida se asocia con pérdida de peso (de 1 a $5 \mathrm{~kg}$ ), sin aumentar el riesgo de hipoglucemia. El riesgo de hipoglucemia en pacientes adultos mayores es más frecuente con el uso de sulfonilureas, Handelsman, et al. (2011).

Ante hiperglucemia posprandial, están indicadas las glinidas o inhibidores de la alfaglucosidasa, o la insulina de corta o rápida duración. La acarbosa reduce la hemoglobina glucosilada entre 0.5 y $1 \%$; sus efectos gastrointestinales adversos son motivo de abandono del tratamiento, Gil, et al. (2013).

La repaglinida es casi tan eficaz como la metformina o la sulfonilurea, para disminuir en 1.5 \% los niveles de HbAlc. La nateglinida es menos eficaz en esta reducción que la repaglinida cuando se utiliza como monoterapia o en terapia combinada. El riesgo de aumento de peso es similar al de 
las sulfonilureas, pero la hipoglucemia puede ser menos frecuente con nateglinida que con algunas sulfonilureas, The National Collaborating Centre for Chronic Conditions, (2008).

Los esquemas de hipoglucemiantes dobles o triples tienen un efecto mayor y reducen la HbA1c más que la monoterapia (reducción absoluta de $1 \%$ ). En pacientes no controlados con sulfonilureas, agregar metformina es más efectivo para el control glucémico que continuar con dosis máximas de sulfonilureas, Ministerio de Sanidad y Consumo, (2008).

La adherencia terapéutica puede definirse como la medida en que el paciente asume las normas o consejos dados por el profesional sanitario, tanto desde el punto de vista de hábitos o estilo de vida recomendados como del propio tratamiento farmacológico prescrito. La falta de adherencia se sitúa entre el 30 y el 51\% en los pacientes con DM tipo 2 (DM2) que toman antidiabéticos orales 6,7, y de cerca del 25\% en pacientes insulinizados, Orozco, et al. (2016).

El apego al tratamiento farmacológico o al control metabólico; la escolaridad y el desconocimiento de la enfermedad fueron las variables estadísticamente significativas para la falta de apego al tratamiento farmacológico, y esto concuerda con lo notificado por otros autores al estudiar enfermedades crónicas.6-8 Sería de suma importancia evaluar los métodos de enseñanza que se imparten en las unidades médicas donde se atiende a pacientes con diagnóstico de diabetes mellitus tipo 2; sobre todo si se considera que esta enfermedad es crónica, y que requiere un control adecuado - con el fin de evitar complicaciones graves a corto y a largo plazo-, en el cual intervienen múltiples disciplinas, sobre todo educativas, y no sólo el apego al tratamiento farmacológico, Durán, et al. (2001). 
Resulta alarmante la baja proporción de pacientes diabéticos controlados encontrados en este análisis que, aún teniendo básicamente una representatividad rural, demuestra que las metas del tratamiento a este grupo de pacientes aún no se han cumplido. El control de la glucemia es de causa multifactorial; se debe mejorar el acceso y la calidad con la que se atiende a este grupo de pacientes e intentar así una mejor adherencia al tratamiento y con ello lograr una menor incidencia de complicaciones secundarias a la enfermedad, padecimientos que representan una gran carga económica tanto para los sistemas de salud del país como para los pacientes y sus familias, Hernández, et al. (2011).

Existe acuerdo en considerar la importancia de la medición periódica del cumplimiento terapéutico, ya que hay distintas modalidades de incumplimiento farmacológico, en función de cuándo decide el paciente dejar de tomar la medicación. Por un lado, es posible que el paciente ni siquiera comience a tomar la medicación, o puede comenzar a tomarla del modo adecuado pero al cabo de un tiempo abandonarla prematuramente. Además, el cumplimiento puede variar en el tiempo, tal y como se ha descrito en otros estudios, observándose un descenso del porcentaje de pacientes cumplidores, que pasa del $82,5 \%$ en los primeros 6 meses de tratamiento al $71,8 \%$ en los últimos 6 meses en pacientes con DM2 que usaban insulina, Márquez, et al. (2012).

El envejecimiento poblacional está condicionando un marcado incremento de la pandemia de diabetes en las personas de edad avanzada. Sin embargo, existen pocas evidencias científicas que apoyen el tratamiento más adecuado de la diabetes en los ancianos. Dada la gran heterogeneidad de la población anciana, que incluye a sujetos con muy diferente capacidad funcional y cognitiva, diversa comorbilidad y con muy diferente expectativa de vida, resulta crucial realizar una valoración global del anciano desde una perspectiva biopsicosocial y abordar integralmente los factores de

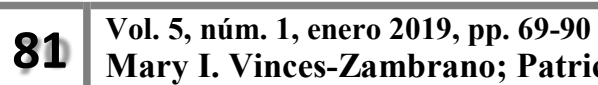
Mary I. Vinces-Zambrano; Patricia M. Espinel-Zambrano; Andrea N. Pico-Tagle; Sarai E. Del Castillo Pavon; Gloria E. Chávez De La Torre; Edwin D. Betancourth-Justicia
} 
riesgo vascular, planteando unos objetivos personalizados de control glucémico, Gómez, et al. (2014).

En ancianos frágiles o con corta expectativa de vida puede ser razonable mantener un objetivo de HbA1c de 7,6-8,5\%. La estrategia terapéutica en el anciano con diabetes tipo 2 debe individualizarse y consensuarse con el paciente y sus cuidadores, en función del objetivo planteado. Mejorar la calidad de vida, preservar la seguridad del paciente y evitar los efectos adversos del tratamiento antidiabético deben ser objetivos básicos. Dada la mayor predisposición de los ancianos a las hipoglucemias y sus graves consecuencias en esta población, deberían priorizarse las terapias antidiabéticas que minimicen el riesgo de episodios hipoglucémicos. Gómez, et al. (2014).

Un $66,4 \%$ de los pacientes alcanzaron el objetivo de control para la HbAlc, un $43,2 \%$ para la PA y un 40,5\% para el colesterol LDL. Se incurrió en IT en un 86,4\% para el colesterol LDL, en un 76,7\% para la PA y en un 40,6\% para la HbAlc. El porcentaje de incumplimiento terapéutico fue del 36,1, 37,5 y 32,0\% para los antidiabéticos, antihipertensivos e hipolipidemiantes, respectivamente. Los pacientes con mayor edad fueron mejor cumplidores. La IT no se relacionó con el CT. No hubo diferencias según sexo en cuanto al grado de control, la IT, la utilización de fármacos, ni el CT, López, et al. (2012).

Los profesionales sanitarios, y especialmente los médicos de atención primaria, deben conocer el fenómeno de la falta de adherencia, detectarlo y averiguar sus causas, así como tener recursos para poder intervenir eficazmente en los pacientes, Orozco, et al. (2016).

En relación a la seguridad y eficacia del uso de terapias herbales y suplementos de vitaminas y minerales tales como el aloe vera, ginseng, nopal y otros, éstos cuentan con diversos estudios y

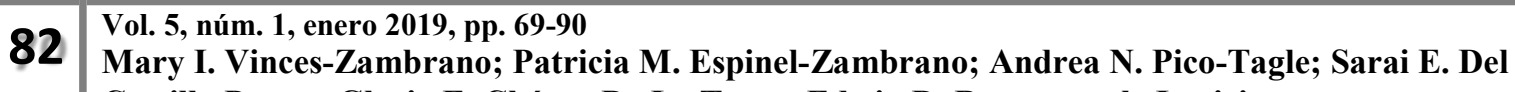
Castillo Pavon; Gloria E. Chávez De La Torre; Edwin D. Betancourth-Justicia
} 
una reciente revisión de más de 100 publicaciones concluyó que la evidencia que permite establecer recomendaciones aún es insuficiente, pero su uso parece ser seguro. Yeh, et al. (2003).

\section{Tratamiento farmacológico del paciente diabético no obeso}

Las sulfonilureas son los fármacos de primera línea cuando no se logran las metas del tratamiento no farmacológico. Su principal mecanismo de acción es reforzar la secreción de la insulina. Los factores que predicen una buena respuesta al medicamento son: 27

- Diagnóstico reciente de DM2.

- Niveles de hiperglucemia de 220 a $240 \mathrm{mg} / \mathrm{dL}$.

- Una función conservada de las células beta del páncreas.

- Pacientes sin antecedentes de insulinoterapia, Oviedo, et al. (2003).

Las sulfonilureas deben añadirse a metformina cuando el control glucémico no sea adecuado. Cuando el tratamiento inicial fue con sulfonilureas por intolerancia a metformina y en caso de no cumplir metas de control glucémico a pesar de utilizar doble terapia oral, se debe utilizar insulina como segundo o tercer fármaco, Ministerio de Sanidad y Consumo, (2008).

Los estudios clínicos no han mostrado la superioridad de una sulfonilurea sobre otra cuando son administradas en la dosis máxima efectiva. Las sulfonilureas son agentes hipoglucemiantes que no tienen efecto directo en los lípidos plasmáticos; es común que el paciente gane peso con su uso, Oviedo, et al. (2003).

Las sulfonilureas están contraindicadas en pacientes con diabetes tipo 1, durante el embarazo y la lactancia, ante complicaciones agudas (cetoacidosis o coma hiperosmolar) y en pacientes

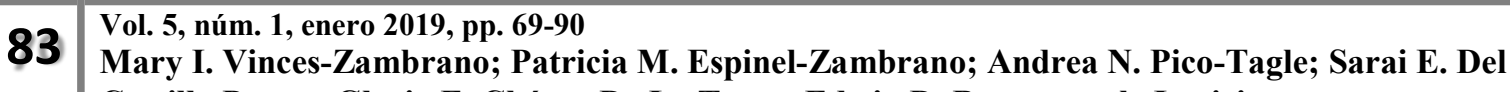
Castillo Pavon; Gloria E. Chávez De La Torre; Edwin D. Betancourth-Justicia
} 
alérgicos a las sulfas. La hipoglucemia es un efecto adverso de este grupo de medicamentos, aunque también se ha reportado dermatosis, discrasias sanguíneas, colestasis, hiponatremia y fenómeno disulfirán. Las sulfonilureas no deberán usarse en pacientes diabéticos obesos de reciente diagnóstico, ya que éstos presentan hiperinsulinemia, Oviedo, et al. (2003).

\section{Tratamiento farmacológico del paciente diabético obeso}

Las biguanidas son los fármacos de primera línea para el tratamiento del paciente obeso El metformin es una biguanida que disminuye los niveles de glucosa plasmática y los niveles de triglicéridos y colesterol, Oviedo, et al. (2003).

El metformin trabaja reduciendo la producción basal de glucosa hepática y reforzando la sensibilidad a la insulina en el músculo. El metformin es el único agente oral antidiabético que cuando es usado en monoterapia ha mostrado una reducción en las complicaciones macrovasculares de la enfermedad, Campbell, Howlett, (1995).

Los efectos adversos más comunes son las alteraciones gastrointestinales, aunque casi siempre son transitorios. La acidosis láctica es una complicación muy rara en los pacientes tratados con el medicamento. No existe evidencia en estudios prospectivos comparativos o de cohortes que el metformin esté asociado con un incremento del riesgo para acidosis láctica, al ser comparado con otros fármacos que reducen la hiperglucemia. Sin embargo, el metformin está contraindicado en aquellas condiciones que favorecen la acidosis láctica, como insuficiencia renal, infecciones graves, insuficiencia cardiaca, insuficiencia hepática, alcoholismo, cirugía mayor, infarto del miocardio, politraumatismo, coma diabético e hipoxia, Misbin, et al. (1998). 
Inhibidores de alfaglucosidasa (acarbosa)

La acarbosa está indicada en pacientes mayores de 60 años con diabetes de reciente inicio y descontrol leve (glucemia entre 126 y $140 \mathrm{mg} / \mathrm{dL}$ ). El principal efecto del medicamento es la disminución de los niveles de la glucosa posprandial. Trabaja al interferir con la digestión de carbohidratos y retrasando la absorción de la glucosa. No provoca ganancia de peso ni afecta los niveles plasmáticos de los lípidos. Los efectos adversos están relacionados con el tracto digestivo; el más común es la flatulencia, que se ha reportado hasta en $30 \%$ de los pacientes, pero el efecto tiende a disminuir al continuar el uso del medicamento, De-Fronzo, (1999).

\section{Insulina}

Debe iniciarse el tratamiento con insulina cuando no se llega a la meta de control glucémico, a pesar del cambio en el estilo de vida y del uso de hipoglucemiantes orales. Es indispensable cerciorarse de la adherencia al cambio del estilo de vida al iniciar la terapia con insulina. Debe explicarse al paciente, desde el momento del diagnóstico, que la insulina es una opción para el control de la diabetes y que puede ser necesaria conforme evoluciona la enfermedad, American Diabetes Association, (2011).

La insulina es el medicamento de elección ante la falla de las dosis máximas de hipoglucemiantes orales. Las insulinas humanas disponibles en nuestro país son las de acción rápida, las de acción intermedia (NPH y lenta) y la insulina lispro. El uso de la insulina de acción rápida no está indicado en el primer nivel de atención, ya que se emplea en el tratamiento de complicaciones metabólicas agudas (cetoacidosis o coma hiperosmolar), o bien, en hiperglucemia secundaria a enfermedades, estrés, diabetes tipo 1 y durante el embarazo, Oviedo, et al. (2003).

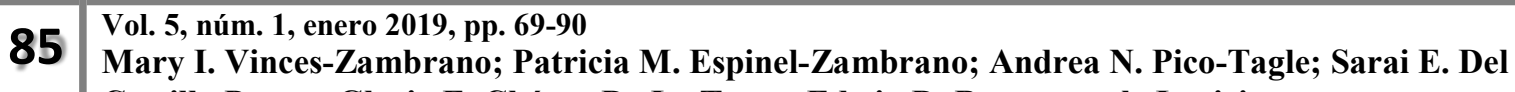
Castillo Pavon; Gloria E. Chávez De La Torre; Edwin D. Betancourth-Justicia
} 
Deberá administrarse insulina basal con análogo de insulina de acción prolongada, más que insulina NPH, por el menor riesgo de hipoglucemia, así como agregar insulina de acción corta prandial cuando el paciente ha logrado la meta de glucemia en ayuno pero no la de HbA1c. Cuando la insulina está indicada para el control de la hiperglucemia posprandial, es preferible utilizar análogos de insulina de acción rápida más que insulina humana regular, debido a que tienen un inicio más rápido y su vida media se asocia con menor riesgo de hipoglucemias. Handelsman, et al. (2011).

La dosis de insulina humana de acción intermedia debe particularizarse para cada paciente. La dosis inicial no debe superar $0.5 \mathrm{UI} / \mathrm{kg}$ de peso. En algunos casos es posible administrar una sola dosis de acción intermedia aplicada por la mañana. Cuando se requieren más de 25 a 30 UI de insulina intermedia, se deberá fraccionar la dosis: dos terceras partes en la mañana y la otra tercera parte en la noche, Oviedo, et al. (2003).

La insulina premezcla (combinación fija de análogos de acción corta y prolongada) puede ser considerada para pacientes que no siguen un régimen de medicamentos; sin embargo, estas preparaciones carecen de flexibilidad en sus componentes y pueden aumentar el riesgo de hipoglucemia en comparación con la insulina basal o con un régimen intensivo, ALAD, (2008).

Para el tratamiento combinado con hipoglucemiantes orales, la dosis de insulina intermedia es de 0.1 a $0.2 \mathrm{UI} / \mathrm{kg}$ de peso, preferentemente aplicada a la hora de acostarse. El automonitoreo (en los casos en que esto sea posible) es de gran utilidad para ajustar la dosis de insulina y alcanzar un buen control metabólico. El médico junto con el equipo multidisciplinario de salud deberá dar la instrucción adecuada al paciente y su familia acerca de las técnicas de aplicación de la insulina, Oviedo, et al. (2003).

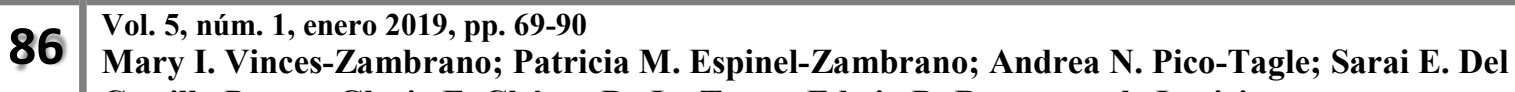
Castillo Pavon; Gloria E. Chávez De La Torre; Edwin D. Betancourth-Justicia
} 


\section{Conclusiones.}

Es alarmante el pobre control en el paciente con diabetes este es un problema para los sistemas de salud por lo que es importante que los médicos en sus prescripciones orienten a los pacientes diabéticos una adherencia terapéutica farmacológica adaptada a cada uno de sus requerimientos con el fin de contribuir al control metabólico y reducir las complicaciones vasculares, acrecentando la calidad y la esperanza de vida de los pacientes, por lo que es fundamental la organización de la asistencia a pacientes diabéticos con el propósito de garantizar el cumplimiento terapéutico de manera suficiente y cual es su importancia con la intención que los pacientes puedan incorporar a su estilo de vida se asocia con una mejoría en la calidad de vida y en el apego terapéutico. La trascendencia económica del consumo inadecuado de fármacos es indiscutible y es un auténtico reto para los administradores, debido al derroche económico que hacen los pacientes. El tratamiento farmacológico en la terapia de la diabetes, concomitantemente con el ejercicio, así, como incorporado al tratamiento de la nutrición.

Sin embargo, es necesario que los profesionales sanitarios, y especialmente los médicos de atención primaria, deben conocer el fenómeno de la falta de adherencia, detectarlo y averiguar sus causas, así como tener recursos para poder intervenir eficazmente en los pacientes mejorar la educación de los profesionales sanitarios que tratan pacientes con diabetes para perfeccionar la prescripción y seguimiento de las pautas del tratamiento farmacológico mismas que deben ser óptimas para cada paciente, según el estado de conocimientos existente en un momento dado.

Es indispensable cerciorarse de la adherencia al cambio del estilo de vida al iniciar la terapia con insulina. Debe explicarse al paciente, desde el momento del diagnóstico, que la insulina es una opción para el control de la diabetes y que puede ser necesaria conforme evoluciona la enfermedad. 


\section{Referencias Bibliográficas}

ALAD, (2008), Guías de diagnóstico, control y tratamiento de la diabetes mellitus tipo 2, Organización Panamericana de la Salud/Asociación Latinoamericana de Diabetes (ALAD), Disponible en http://www.paho.org/spanish/ad/dpc/nc/dia-guia-alad.pdf

American Diabetes Association, (2011), Standars of Medical Care in Diabetes-2011, Diabetes Care., 34 (Suppl 1): S11-61.

American Diabetes Association, (2002), Standards of medical care for patients with diabetes mellitus, Diabetes Care 2002, 25: S33-S49.

Campbell, I., Howlett, H., (1995), Worldwide experience of metformin as an effective glucoselowering agent: a meta-analysis, Diabetes Metab Rev. 11 (Suppl): S57-S62.

Cramer, J., Benedict, A., Muszbek, N., Keskinaslan, A., Khan, Z., (2008), The significance of compliance and persistence in the treatment of diabetes, hypertension and dyslipidaemia: a review. Int J Clin Pract.; 62: 76---87.

De-Fronzo, R., (1999), Pharmacologic therapy for type 2 diabetes mellitus, (Review), Ann Inter Med 1999, 131 (4): 281-303.

Donnelly, L., Morris, A., Evans, J., (2007), Adherence to insulin and its association with glycaemic control in patients with type 2 diabetes, QJM., DARTS/MEMO collaboration, 100: 345---50.

Durán, B., Rivera, B., Franco, E., (2001), Apego al tratamiento farmacológico en pacientes con diagnóstico de diabetes mellitus tipo 2, Salud Publica de México, Vol.43, № 3: 233-236.

Evans, J., Donnan, P., Morris, A., (2002), Adherence to oral hypoglycaemic agents prior to insulin therapy in Type 2 diabetes. Diabet Med. 19(8): 685-688.

Freire WB., Ramírez-Luzuriaga MJ., Belmont P., Mendieta MJ., Silva-Jaramillo MK., Romero N., Sáenz K., Piñeiros P., Gómez LF., Monge R. (2014). Tomo I: Encuesta Nacional de Salud y Nutrición de la población ecuatoriana de cero a 59 años. ENSANUT-ECU 2012. Ministerio de Salud Pública/Instituto Nacional de Estadísticas y Censos. Quito-Ecuador

Gil, L., Sil, M., Domínguez, E., Torres, L., Medina, J., (2013), Diagnóstico y tratamiento de la diabetes mellitus tipo 2, Revista Médica del Instituto Mexicano de Seguro Social, 51(1): 10419.

Goldstein, D., Little, R., Lorenz, R., Malone, J., Nathan, D., Peterson, Ch., Sacks D., (2004), Tests of glycemia in diabetes. Diabetes Care 2004; 27(7): 1761-1773.

Gómez, R., Díez, J., Formiga, F., Lafita, J., Rodríguez, L., González, E., Menéndez, E., Sangrós, J., (2014), Tratamiento de la diabetes tipo 2 en el paciente anciano, Medicina Clínica, Volume 142, Issue 2, Pages 89-90. https://doi.org/10.1016/j.medcli.2012.10.003Get rights and content 
Handelsman, Y., Mechanick, J., Blonde, L., Grunberger, G., Bloomgarden, Z., Bray, G., et al. (2011), Medical Guidelines for clinical practice for developing a diabetes mellitus comprehensive care plan: executive summary, American Association of Clinical Endocrinologists, Endocr Pract. 2011, 17(2): 287-302.

Hernández, A., Elnecavé, A., Huerta, N., Reynoso, N., (2011), Análisis de una encuesta poblacional para determinar los factores asociados al control de la diabetes mellitus en México, Salud Publica México, Vol. 53, № 1: 34-39.

Kim, H., Oh, J., (2003), Adherence to diabetes control recommendations: impact of nurse telephone calls. J Adv Nurs 2003, 44(3): 256-261.

López, F., Brotons, C., Moral, I., Cols, C., Selva, A., Aguado, A., Miravet, S., (2012), Inercia y cumplimiento terapéutico en pacientes con diabetes mellitus tipo 2 en atención primaria, Medicina Clínica, Volume 138, Issue 9, Pages 377-384.

https://doi.org/10.1016/j.medcli.2011.07.023Get rights and content

Márquez, E., Martell, N., Gil, V., Casado, J., Martín, J., Ferraro, J., Chaves, R. y Fernández, A., (2012), El cumplimiento terapéutico con insulina en el tratamiento de la diabetes mellitus tipo 2: estudio CUMINDIAB, Atención Primaria. 2012, 44(2): 74---81. www.elsevier.es/ap

Márquez Contreras, E., Casado Martínez, JJ., (2001), El incumplimiento farmacológico en las enfermedades cardiovasculares. Med Clin., 116 (Supl 2): 546.

Martín, F., Rodríguez, M., (2001), Apoyo familiar en el apego al tratamiento de la hipertensión arterial esencial, Salud Publica Mex 2001, 43: 336-339.

Matsuoka, K., (2001), A diabetes care team-role of diabetes specialists and certified diabetes educator, Jpn J Clin Pathol 2001, 49(12): 1212-1217.

Ministerio de Sanidad y Consumo, (2008), Guía de práctica clínica sobre diabetes tipo 2, Ministerio de Sanidad y Consumo, España.

Misbin, R., Green, L., Stadel, B., Guerguian, J., Gubbi, A., Fleming, G., (1998), Lactic acidosis in patients treated with metformin, N Engl J Med. 338: 265-266.

Orozco, D., Mata, M., Artola, S., Conthe, P., Mediavilla, J. y Miranda, C., (2016), Abordaje de la adherencia en diabetes mellitus tipo2: situación actual y propuesta de posibles soluciones, Atención Primaria.2016, 48(6): 406---420 www.elsevier.es/ap

Ortiz, M., Ortiz, E., (2007), Psicología de la salud: Una clave para comprender el fenómeno de la adherencia terapéutica, Rev Méd Chile 2007; 135: 647-652.

Oviedo, M., Espinosa, F., Reyes, H., Trejo, J., Gil, E., (2003), Guía clínica para el diagnóstico y tratamiento de la diabetes mellitus tipo 2, Revista Médica del IMSS, Volumen 41 Suplemento 1, 527-546. 
Rush, W., O’Connor, P., (2002), Validation of an automated medication adherence measure for diabetes patients, Diabetes 2002, 51(Suppl 2): A76.

The National Collaborating Centre for Chronic Conditions, (2008), Chronic Kidney Disease. National clinical guideline for early identification and management in adults in primary and secondary care, London, UK: Royal College of Physicians.

Vinagre, I., Mata, M., Hermosilla, E., Morros, R., Fina, F., Rosell, M., et al. (2012), Control of glycemia and cardiovascular risk factors in patients with type 2 diabetes in primary care in Catalonia (Spain), Diabetes Care, 2012, 35: 774---9.

WHO, (2013), World Health Organization, Diabetes Fact sheet, No. 312, Updated March, Disponible en: http:// www.who.int/mediacentre/factsheets/fs312/en/index.html

Yeh G., Eisenberg D., Kaptchuk T., Phillips R., (2003), Systematic Review of Herbs and Dietary Supplements for Glycemic Control in Diabetes, Diabetes Care 2003; 26: 1277-1294. 